\title{
Rare GABRA3 variants are associated with epileptic seizures, encephalopathy and dysmorphic features
}

\author{
Cristina Elena Niturad, ',* Dorit Lev, 2,3,4,* Vera M. Kalscheuer, ${ }^{5,6, *}$ Agnieszka Charzewska, ${ }^{7}$ \\ Julian Schubert, ${ }^{1,8}$ Tally Lerman-Sagie, ${ }^{3,4,9}$ Hester Y. Kroes, ${ }^{10}$ Renske Oegema, ${ }^{10}$ \\ Monica Traverso, " Nicola Specchio, ${ }^{12}$ Maria Lassota, ${ }^{13}$ Jamel Chelly, ${ }^{14}$ \\ Odeya Bennett-Back, ${ }^{15}$ Nirit Carmi, ${ }^{3,4,10}$ Tal Koffler-Brill, ${ }^{16}$ Michele lacomino, " I \\ Marina Trivisano, ${ }^{12}$ Giuseppe Capovilla, ${ }^{17}$ Pasquale Striano, $^{18}$ Magdalena Nawara, $^{7}$ \\ Sylwia Rzońca, ${ }^{7}$ Ute Fischer, ${ }^{5,6}$ Melanie Bienek, ${ }^{5}$ Corinna Jensen, ${ }^{5, \$}$ Hao Hu, ${ }^{5,8}$ \\ Holger Thiele, ${ }^{19}$ Janine Altmüller, ${ }^{19,20}$ Roland Krause, ${ }^{8}$ Patrick May, ${ }^{8}$ Felicitas Becker, \\ EuroEPINOMICS Consortium, Rudi Balling, ${ }^{8}$ Saskia Biskup, ${ }^{21}$ Stefan A. Haas, ${ }^{22}$ \\ Peter Nürnberg, ${ }^{19}$ Koen L. I. van Gassen, ${ }^{10}$ Holger Lerche,' Federico Zara, ${ }^{1, *}$ \\ Snezana Maljevic ${ }^{1, *, \phi}$ and Esther Leshinsky-Silver ${ }^{2,3,16, *, \dagger}$
}

*These authors contributed equally to this work.

${ }^{\dagger}$ Deceased.

Genetic epilepsies are caused by mutations in a range of different genes, many of them encoding ion channels, receptors or transporters. While the number of detected variants and genes increased dramatically in the recent years, pleiotropic effects have also been recognized, revealing that clinical syndromes with various degrees of severity arise from a single gene, a single mutation, or from different mutations showing similar functional defects. Accordingly, several genes coding for $\mathrm{GABA}_{\mathrm{A}}$ receptor subunits have been linked to a spectrum of benign to severe epileptic disorders and it was shown that a loss of function presents the major correlated pathomechanism. Here, we identified six variants in GABRA3 encoding the $\alpha_{3}$-subunit of the GABA receptor. This gene is located on chromosome Xq28 and has not been previously associated with human disease. Five missense variants and one microduplication were detected in four families and two sporadic cases presenting with a range of epileptic seizure types, a varying degree of intellectual disability and developmental delay, sometimes with dysmorphic features or nystagmus. The variants co-segregated mostly but not completely with the phenotype in the families, indicating in some cases incomplete penetrance, involvement of other genes, or presence of phenocopies. Overall, males were more severely affected and there were three asymptomatic female mutation carriers compared to only one male without a clinical phenotype. X-chromosome inactivation studies could not explain the phenotypic variability in females. Three detected missense variants are localized in the extracellular GABA-binding $\mathrm{NH}_{2}$-terminus, one in the M2-M3 linker and one in the M4 transmembrane segment of the $\alpha_{3}$-subunit. Functional studies in Xenopus laevis oocytes revealed a variable but significant reduction of GABA-evoked anion currents for all mutants compared to wild-type receptors. The degree of current reduction correlated partially with the phenotype. The microduplication disrupted GABRA3 expression in fibroblasts of the affected patient. In summary, our results reveal that rare loss-of-function variants in GABRA3 increase the risk for a varying combination of epilepsy, intellectual disability/developmental delay and dysmorphic features, presenting in some pedigrees with an X-linked inheritance pattern.

Received March 15, 2017. Revised July 10, 2017. Accepted July 26, 2017. Advance Access publication October 7, 2017 (C) The Author (2017). Published by Oxford University Press on behalf of the Guarantors of Brain. All rights reserved.

For Permissions, please email: journals.permissions@oup.com 
1 Department of Neurology and Epileptology, Hertie Institute for Clinical Brain Research, University of Tübingen, Tübingen, Germany

2 Institute of Medical Genetics, Wolfson Medical Center, Holon, Israel

3 Metabolic-Neurogenetic Clinic, Wolfson Medical Center, Holon, Israel

4 Sackler School of Medicine, Tel-Aviv University, Tel-Aviv, Israel

5 Department of Human Molecular Genetics, Max Planck Institute for Molecular Genetics, Berlin, Germany

6 Research Group Development and Disease, Max Planck Institute for Molecular Genetics, Berlin, Germany

7 Institute of Mother and Child, Department of Medical Genetics, Kasprzaka 17A, 01-211 Warsaw, Poland

8 Luxembourg Centre for Systems Biomedicine, University of Luxembourg, Luxembourg

9 Pediatric Neurology Unit, Wolfson Medical Center, Holon, Israel

10 Department of Genetics, University Medical Center Utrecht, 3508 AB, The Netherlands

11 Laboratory of Neurogenetics and Neuroscience, Institute G. Gaslini, Genova, Italy

12 Neurology Unit, Bambino Gesù Children's Hospital, IRCCS, Rome, Italy

13 Genetic Clinic, Hetmańska 21, 35-045 Rzeszów, Poland

14 IGBMC, Strasbourg, France

15 Pediatric Neurology Unit, Hadassah Medical Center, Jerusalem

16 Molecular Genetics Laboratory, Wolfson Medical Center, Holon, Israel

17 Epilepsy Center, Department of Child Neuropsychiatry, C. Poma Hospital, Mantova, Italy

18 Pediatric Neurology and Muscular Diseases Unit, Department of Neurosciences, Rehabilitation, Ophtalmology, Genetics, Maternal and Child Health, Institute G. Gaslini, Genoa, Italy

19 Cologne Center for Genomics, Universität zu Köln, Köln, Germany

20 Institute of Human Genetics Universitätsklinik, Köln, Germany

21 CeGaT GmbH, Tübingen, Germany

22 Department of Computational Molecular Biology, Max Planck Institute for Molecular Genetics, Berlin, Germany

"Present address: Institut für Funktionelle Genomforschung, Universitätsmedizin Greifswald, Greifswald, Germany

${ }^{\$}$ Present address: Guangzhou Institute of Pediatrics, Guangzhou Women and Children's Medical Center, China

${ }^{\phi}$ Present address: The Florey Institute of Neuroscience and Mental Health, Melbourne, Australia

Correspondence to: Holger Lerche

Department of Neurology and Epileptology, Hertie Institute for Clinical Brain Research, University of Tübingen, Tübingen, Germany E-mail: holger.lerche@uni-tuebingen.de

Keywords: epilepsy; intellectual disability; X-linked disease; neuronal inhibition

Abbreviation: GGE = genetic generalized epilepsy

\section{Introduction}

Genetic epilepsy has been commonly associated with alterations in genes coding for ion channels and receptors (Lerche et al., 2013). Recent employment of next generation sequencing techniques, including exome sequencing and whole genome copy number variant analysis, corroborate such observations by identifying a number of novel ion channel and receptor gene variants in epilepsy patients (Veeramah et al., 2012; Weckhuysen et al., 2012; Epi4K Consortium et al., 2013; Carvill et al., 2014; Lemke et al., 2014; Nava et al., 2014; Schubert et al., 2014; Weckhuysen and Korff, 2014; Larsen et al., 2015; Syrbe et al., 2015; Leu et al., 2016; Papandreou et al., 2016; Møller et al., 2017). Apart from identifying novel disease genes, these findings reveal several additional developments. First, they expand the range of syndromes associated with some of the previously identified epilepsy genes. Second, they suggest that severe epileptic encephalopathies, encompassing both refractory seizures and developmental delay often represent sporadic cases, with de novo occurring mutations. Lastly, they point to an increasing number of genes and variants showing a pleiotropic effect, when distinct clinical syndromes with different degrees of severity arise from a single gene, a single variant, or from different variants showing similar functional defects (Claes et al., 2001; Harkin et al., 2007; Veeramah et al., 2012; Weckhuysen et al., 2012; Epi4K Consortium et al., 2013; Suls et al., 2013; Carvill et al., 2014; Nava et al., 2014; Weckhuysen and Korff, 2014; Blanchard et al., 2015; Howell et al., 2015; Syrbe et al., 2015; Epi4K Consortium, 2016; Johannesen et al., 2016; Janve et al., 2017; Møller et al., 2017; Shen et al., 2017; Wolff et al., 2017).

GABA is the main inhibitory neurotransmitter in the adult brain. Its action depends on two classes of proteins, ionotropic $\mathrm{GABA}_{\mathrm{A}}$ and metabotropic $\mathrm{GABA}_{\mathrm{B}}$ receptors, which mediate fast and slow synaptic inhibition, respectively. Furthermore, both receptor types are involved in tonic inhibition. Nineteen different genes from eight different classes encode $\mathrm{GABA}_{\mathrm{A}}$ receptor subunits, which combine into heteropentamers to form postsynaptic ligandgated anion channels. Pentamers usually contain two alpha and two beta subunits, which conjoin with a subunit 
from one of the other classes. Subunit combination determines functional properties of the receptor as well as its spatiotemporal expression pattern (Fritschy and Panzanelli, 2014).

Presuming that neuronal hyperexcitability seen in epilepsy arises from a disturbed balance of inhibitory and excitatory neurotransmission in the brain, it is somewhat surprising that so far only four genes (GABRA1, GABRB3, GABRG2 and GABRD) encoding the $\alpha_{1^{-}}, \gamma_{2^{-}}$, $\beta_{3^{-}}$and $\delta$-subunits of $\mathrm{GABA}_{\mathrm{A}}$ receptors have been directly associated with epilepsy (Macdonald et al., 2012). Initially, the correlated syndromes included childhood absence epilepsy, juvenile myoclonic epilepsy, and febrile seizures with or without epilepsy including generalized/genetic epilepsy with febrile seizures plus (GEFS +) (Baulac et al., 2001; Wallace et al., 2001; Cossette et al., 2002; Harkin et al., 2002; Kananura et al., 2002; Maljevic et al., 2006; Macdonald et al., 2012). Recent studies extended this list to different forms of epileptic encephalopathies, including epilepsy with myoclonic-atonic seizures and Dravet syndrome (Carvill et al., 2014; Epi4K Consortium, 2016; Johannesen et al., 2016; Papandreou et al., 2016; Janve et al., 2017; Møller et al., 2017; Shen et al., 2017). The association of a single gene with a spectrum of partially overlapping epileptic phenotypes, from mild to severe ones has been mainly attributed to the brain sodium channel gene SCN1A and to SLC2A1 encoding the GLUT1 transporter (Claes et al., 2001; Harkin et al., 2007; Leen et al., 2010; Lerche et al., 2013; Weckhuysen and Korff, 2014). However, a similar genotype-phenotype correlation pattern has recently also been reported for the GABRA1, GABRB3 and GABRG2 genes encoding $\alpha_{1^{-}}, \beta_{3^{-}}$and $\gamma_{2^{-}}$ subunits of the $\mathrm{GABA}_{\mathrm{A}}$ receptor, respectively (Carvill et al., 2014; Epi4K Consortium, 2016; Johannesen et al., 2016; Kang and Macdonald, 2016; Møller et al., 2017).

In this study, we identified several variants in the GABRA3 gene, encoding the $\alpha_{3}$-subunit of $\mathrm{GABA}_{\mathrm{A}}$ receptors, in families and sporadic cases affected either by variably severe epilepsy with encephalopathy, or by genetic generalized epilepsy (GGE), presenting a milder form of epilepsy with complex inheritance.

\section{Materials and methods}

\section{Patients and cohorts}

Parents or the legal guardian of each patient enrolled in this study signed an informed consent form for participation. The study was approved by the local ethics committees of each participating clinical centre. Genomic DNA of the individuals was extracted from peripheral blood according to standard procedures.

The analysed cohorts included: (i) a cohort from the Wolfson Medical Center, Holon, Israel wih 15 families with various forms of epilepsy; (ii) a cohort of 480 families with Xlinked intellectual disability (XLID) collected by the EUROMRX consortium and associated groups; (iii) a cohort of
600 cases undergoing diagnostic high-density array-comparative genomic hybridization (CGH) screening; and (iv) a cohort of 238 exome-sequenced cases with GGE collected by the EuroEPINOMICS consortium. Additionally, diagnostic services within the European epilepsy community were also included. Detailed description of the patient cohorts is provided in the Supplementary material.

\section{Whole exome sequencing}

\section{Target enrichment}

Whole exome sequencing and target enrichment were performed according to standard procedures at three different centres [Wolfson Medical Center (WMC), Holon, Israel; CeGaT GmbH, Tübingen, Germany; Cologne Center for Genomics (CCG), Cologne, Germany]. Samples were enriched with Agilent Sureselect Human All Exon v.2 (WMC), or v.5 (CeGaT) or v.6 (CCG) kit. Sequencing was carried out on Illumina HiSeq2000 or HiSeq2500 platforms as 100 bp pairedend runs (WMC, Cegat) or 76 bp paired-end runs (CCG).

\section{Data processing}

Adaptors were removed with Skewer 0.1.1161 (Cegat) or cutadapt (CCG). The data preprocessing and variant calling was performed following the GATK (DePristo et al., 2011) best practice or the Varbank pipeline v.2.3 developed by the Cologne Center of Genomics, University Cologne. For alignment of the reads, we used bwa-mem (Li and Durbin, 2009) with default parameters and hg19 as reference. The sam file format was converted to bam files with samtools ( $\mathrm{Li}$ et al., 2009). The subsequent steps by means of sorting bam files, marking duplicated reads and addition of read groups was performed with default parameters using picard tools (https:// github.com/broadinstitute/picard). In order to recalibrate base quality scores and do local realignment, GATK version 3.2 was used. Variant filtering was done using GATK best practice (for SNV: FS $>60.0$, QD $<2.0, \mathrm{MQ}<40.0$, ReadPosRank Sum $<-8.0$, MQRankSum $<-12.5$, DP $<10.0$, GQ_MEAN $<20.0$, VQSLOD $<0$, ABHet $>0.75$ or $<0.25$; For Indel: QD $>2.0$, FS $>200$, ReadPosRankSum $<-20.0$, DP $<10.0$ ).

\section{Variant annotation}

The variants were annotated using ANNOVAR (Wang et al., 2010) (WMC, CeGaT, CCG) or additionally by in-house software (CCG). RefSeq, dbNSFP30a (Liu et al., 2011, 2016), caddgt20 (Kircher et al., 2014), ClinVar (20150330), HGMD (1000 Genomes Project Consortium et al., 2012), 1000 Genomes, dbSNP (Sherry et al., 2001), ExAC (Lek et al., 2016) (release 0.3) and the EVS (Exome Variant Server, NHLBI GO Exome Sequencing Project (ESP), Seattle, WA (URL: http://evs.gs.washington.edu/EVS/) were used. Noncoding variants and variants with minor allele frequency (MAF) $<0.01$ were filtered out. In addition, variants were filtered against an in-house database to exclude pipeline-related artefacts $(\mathrm{MAF}<0.02)$. Only variants with a coverage $\geqslant 10 \times$ were analysed. All genes were filtered for the expression in brain and variants not being located in the repeat regions (Reumers et al., 2011).

Furthermore, we filtered variants to be deleterious when one of the following criteria was fulfilled: the variant had to be (i) predicted by 5 of 11 missense prediction scores (SIFT, 
Polyphen_HDiv, Polyphen_HVar, LRT, MutationTaster, MutationAssessor, FATHMM, MetaSVM, MetaLR, VEST3, Provean); (ii) scored in caddgt20; (iii) pathogenic in ClinVar; (iv) in HGMD; and ( $v$ ) highly conserved in two conservation tools (GERP $++\mathrm{RS} \geqslant 3, \quad$ PhyloP $\geqslant 0.95$ and Siphy $\geqslant 10$ ). Nonsense, frameshift and splicing variants were included per se.

\section{$\mathrm{X}$-chromosome exome sequencing}

To identify the disease-causing mutations in families with $\mathrm{X}$ linked intellectual disability we performed X-chromosome exome sequencing using DNA of the index patients and analysed the data, as previously described (Hu et al., 2014, 2016).

\section{Diagnostic panels}

After referral for routine diagnostic exome sequencing, exomes of a child and their parents (Families 4 and 5) were enriched using the Agilent SureSelect XT Human All Exon kit V5 (Family 5) and V6 (Family 4) and sequenced in rapid $2 \times 100$ bp run mode on the HiSeq2500 sequencing system (Illumina) at a mean target depth of $100 \times$. The target was defined as all coding exons of UCSC and Ensembl $\pm 20 \mathrm{bp}$ intron flanks. At this depth $>95 \%$ of the target is covered at least $15 \times$. Reads were aligned to hg19 using BWA (BWA-MEM v0.7.5a) and variants were called using the GATK haplotype caller (v2.7-2 and v3.4.46). Detected variants were annotated, filtered and prioritized using the Bench NGS Lab platform (Agilent-Cartagenia). Analysis was based on a tiered analysis approach. In the first tier known intellectual disability genes were analysed. This first tier did not lead to a diagnosis. The second tier, which filters for de novo variants, resulted in the detection of the de novo GABRA3 variants. The last tier, which filters for recessive variants, did not result in additional candidates.

\section{Microduplication analysis}

Array-CGH was performed on $44 \mathrm{k}$ slide (Agilent) as previously described (Coppola et al., 2010). Assays showing a DLRS (derivative of $\log$ ratio spread) score $>0.3$ were excluded. Detection of gains and losses was performed using the ADM-2 algorithm with a moving average of $500 \mathrm{~kb}$ and a threshold of 6.0. To further define genomic breakpoints on Patient II-2 of Family 3, a high-density 244K microarray was used according to the same protocol. To assess the effect of the microduplication on GABRA3 transcription we amplified GABRA3 exons $1-2$ and $8-9$ by RT-PCR using retrotranscribed RNA from fibroblasts of the proband and of a sexmatched control (iScript ${ }^{\mathrm{TM}}$ cDNA synthesis kit, Bio-Rad Laboratories).

\section{Validation of GABRA3 variants and segregation analysis}

For validation of the GABRA3 variants identified in this study and for segregation analysis we used gene-specific primers flanking the exons that harbour the mutations and determined genotypes for all available family members by conventional Sanger sequencing of specific PCR products. Primer sequences are available upon request.

\section{$\mathrm{X}$-inactivation test}

To assess the inactivation status of chromosome $\mathrm{X}$ in heterozygous female carriers of GABRA3 variants we used the human androgen receptor (HUMARA) assay as described (Gibson et al., 2005).

\section{Functional investigations}

\section{Mutagenesis and RNA preparation}

We used the QuikChange ${ }^{\circledR}$ kit (Stratagene) to engineer five missense mutations, p.T166M, p.Q242L, p.T336M, p.Y474C and p.G47R in the $\mathrm{GABA}_{\mathrm{A}}$ receptor $\alpha_{3}$-subunit cDNA (NM_000808) cloned in the pcDNA3 (kind gift from Dr Steven Petrou, Melbourne). All mutations were confirmed and additional changes excluded by Sanger sequencing. Primers are available upon request. cRNA was prepared using the T7 RNA polymerase kit from Roche.

\section{Oocyte preparation and injection}

The use of animals and all experimental procedures were approved by local authorities (Regierungspräsidium Tübingen, Tübingen, Germany). Oocytes were obtained from the Institute of Physiology I, Tübingen, or purchased from EcoCyte Bioscience. Preparation of oocytes for recordings included treatment with collagenase $(1 \mathrm{mg} / \mathrm{ml}$ of type CLS II collagenase, Biochrom) in OR-2 solution (in $\mathrm{mM}: 82.5 \mathrm{NaCl}$, $2.5 \mathrm{KCl}, 1 \mathrm{MgCl}_{2}$ and 5 HEPES, $\mathrm{pH} 7.6$ ), followed by thorough washing and storing at $16^{\circ} \mathrm{C}$ in Barth solution (in $\mathrm{mM}$ : $88 \mathrm{NaCl}, 2.4 \mathrm{NaHCO}_{3}, 1 \mathrm{KCl}, 0.41 \mathrm{CaCl}_{2}, 0.82 \mathrm{MgSO}_{4}$ and 5 Tris/ $\mathrm{HCl}, \mathrm{pH} 7.4$ with $\mathrm{NaOH}$ ) supplemented with $50 \mu \mathrm{g} / \mathrm{ml}$ gentamicin (Biochrom). To compare current amplitudes of wild-type and mutant channels, the same amounts of cRNA were injected on the same day using the same batch of oocytes plated in 96 well-plates and measured in parallel at Days 1-3 after injection. The combination used was $\alpha_{3} \beta_{2} \gamma_{2}$ s in a 1:1:2 ratio. All cRNA concentrations were adjusted to $2 \mu \mathrm{g} / \mu \mathrm{l}$ and $70 \mathrm{nl}$ of the corresponding cRNA was injected using Roboinject $^{\circledR}$ (Multi Channel Systems).

\section{Automated oocyte two-microelectrode voltage clamp}

GABA-evoked currents in oocytes were recorded at room temperature $\left(20-22^{\circ} \mathrm{C}\right)$ using Roboocyte $2^{\circledR}$ (Multi Channel Systems). Pre-pulled and prepositioned intracellular glass microelectrodes had a resistance of $0.3-1 \mathrm{M} \Omega$ when filled with $1 \mathrm{M} \mathrm{KCl} / 1.5 \mathrm{M} \mathrm{KAc}$. The bath solution was ND96 (in mM: $93.5 \mathrm{NaCl}, 2 \mathrm{KCl}, 1.8 \mathrm{CaCl}_{2}, 2 \mathrm{MgCl}_{2}, 5 \mathrm{HEPES}, \mathrm{pH}$ 7.5). Currents were sampled at $1 \mathrm{kHz}$. GABA-evoked currents were used to analyse the activation of the wild-type using automated two-microelectrode voltage clamping. Different GABA concentrations (in $\mu \mathrm{M}: 1,3,10,40,100,300,1000$ ) diluted in ND96 solution were applied for $15 \mathrm{~s}$ to activate the channels. The holding potential was $-70 \mathrm{mV}$.

\section{Electrophysiological data analysis}

The amplitude of the GABA-induced currents was analysed using Roboocyte2+ (Multi Channel Systems), Clampfit (pClamp 8.2, Axon Instruments), Microsoft Excel (Microsoft, Redmond, WA) and Graphpad Prism software (GraphPad Software). Current response for each GABA concentration 
was normalized to the maximum response evoked by the highest GABA concentration. The normalized GABA responses of each cell were fitted to the four-parameter logistic equation:

$$
\mathrm{Y}=\min +\frac{(\max -\min )}{1+10^{((\log \mathrm{EC} 50-\mathrm{X}) * \mathrm{nH})}}
$$

with max and min being the maximum and minimum evoked responses and $\mathrm{X}$ the corresponding GABA concentration. The $\mathrm{EC}_{50}$ value is the concentration of the agonist at which half of the maximum response is achieved while the $\mathrm{nH}$ presents the Hill coefficient, which determines the steepness of the dose response curve. $\mathrm{EC}_{50}$ values were determined for each oocyte and the averaged values for wild-type and each mutation are shown as mean \pm standard error of the mean (SEM). Current amplitudes recorded in parallel on the same experimental day in response to $1 \mathrm{mM}$ GABA application from oocytes expressing wild-type or mutant channels, were normalized to the mean value of the wild-type response.

\section{Western blot analysis}

Injected Xenopus oocytes expressing either wild-type or mutant $\alpha_{3} \beta_{2} \gamma_{2 s}$ receptors were lysed in a buffer containing $20 \mathrm{mM}$ Tris, $100 \mathrm{mM} \mathrm{NaCl}, 1 \mathrm{mM}$ EDTA, $0.5 \%$ Triton $^{\mathrm{TM}} \mathrm{X}-100$ and $10 \%$ glycerol with cOmplete protease inhibitors (Roche). Water-injected oocytes were used as a control. Protein concentration was measured (DC Protein Assay, Bio-Rad) and $25 \mu \mathrm{g}$ of protein was separated using SDS-PAGE on $8 \%$ polyacrylamide gels. After the transfer onto nitrocellulose membrane (Protran $^{\circledR}$, Whatman), protein detections were performed using a rabbit polyclonal antibody against the $\mathrm{GABA}_{\mathrm{A}}$ receptor $\alpha_{3}$-subunit (1:250; HPA000839 Sigma) and against actin (1:1000; Sigma A3853) as a loading control. Quantification of signals was performed using ImageJ software (NIH). Expression levels were normalized to actin and pooled from four different experiments.

\section{Statistical analysis}

Data were tested for normal distribution using GraphPad Prism 6 (GraphPad Software). Groups were compared using one-way ANOVA with Dunnett's post hoc test for normally distributed data or one-way ANOVA on ranks with Dunn's post hoc test for not normally distributed data. All data are presented as mean \pm SEM. Statistical differences are indicated in the figure legends using the following symbols: ${ }^{*} P<0.05$, $\because P<0.001, * * P<0.0001$.

\section{Results}

The study was initiated by recruitment of 15 families with various forms of epilepsy (Supplementary material), including Family 1 of Israeli Jewish origin, in which the disease status of affected individuals was indicative of an X-linked inheritance. Two males in this pedigree (Fig. 1A) were severely affected with pharmacoresistant epileptic encephalopathies with infantile or childhood onset, featuring infantile spasms, tonic and generalized tonic-clonic seizures, moderate-to-severe intellectual disability and developmental delay




with speech problems. In contrast, two affected females had a much milder phenotype, which included well treatable generalized tonic-clonic seizures and mild learning disability. All of them had micrognathia, short stature and further dysmorphic features (Fig. 1B), as well as nystagmus. Additionally, cleft palate was present in all but Patient III-2 (Table 1 and Supplementary material). Whole exome sequencing in Patient III-1 of this family (Fig. 1A) revealed a variant in GABRA3 as the most plausible disease causing change identified on chromosome X (c.725A > T, p.Q242L, Fig. 1C) (Supplementary material). The p.Q242 is a highly conserved amino acid (Fig. 1D). The variant is predicted to be deleterious according to PolyPhen-2, SIFT and MutationTaster, and is found neither in the ExAC (exome aggregation consortium) database nor in gnomAD (genome aggregation database) (Supplementary Table 1). Direct sequencing in the whole family revealed perfect cosegregation showing that all affected individuals (mother, two affected sons, and daughter) carry the same p.Q242L variant, whereas the healthy son, grandmother, maternal aunts and uncle do not carry this variant (Fig. 1A). To exclude further disease-associated variants, we performed exome sequencing of the remaining three affected individuals and the healthy brother. Among the variants shared by the affected, but not the healthy individual, we found no particular changes in genes that had been previously associated with dysmorphic features, intellectual disability or epilepsy (Supplementary Table 2). We thus assume that the p.Q242L variant is pathogenic and mainly responsible for all clinical features observed in this family.

To further assess the role of GABRA3 in epilepsy and intellectual disability, we selected three clinical cohorts according to the main clinical features of Family 1: a cohort of 480 families with X-linked intellectual disability (XLID) collected by the EURO-MRX consortium and associated groups, a cohort of 600 cases undergoing diagnostic highdensity array-CGH screening, and an exome-sequenced cohort of 238 cases with idiopathic/genetic generalized epilepsy (IGE/GGE) collected by the EuroEPINOMICS consortium. Additionally, diagnostic services in our European epilepsy community were included in our search for pathogenic variants in GABRA3.

$\mathrm{X}$-chromosome exome resequencing of index patients from 480 families with XLID, performed as previously described (Hu et al., 2014, 2016), identified a likely deleterious GABRA3 missense variant in Family 2, corroborating the findings for Family 1 . Segregation analysis performed using conventional Sanger sequencing revealed the presence of the variant in four males who are first degree cousins (Fig. 2A). Their maternal uncle (Patient II:1), who has intellectual disability, is not a mutation carrier and also presented with a different haplotype. Moreover, four mildly affected females from this family carry this variant (Fig. 2A). The index proband (Patient III:10, Fig. 2A and B) and his brother (Patient III:13) have mild intellectual disability and delayed speech. Both suffered from generalized tonic-clonic seizures and their mother (Patient II:6) suffered from absences. Their sister (Patient III:12) carries the variant and has learning problems. The two other male mutation carriers in another branch of the family have different phenotypes-one of them has intellectual disability (Patient III:7) and the other is unaffected (Individual III:6). Their mother, who is a mutation carrier, suffered from absence seizures. Another affected male in the family (Patient III:4) also has intellectual disability but was unavailable for molecular testing. Recent clinical re-evaluation of the proband and his brother revealed dysmorphic features similar to those seen in affected individuals of Family 1 (e.g. nystagmus, micrognathia, arched palate) (Table 1 and Supplementary material). However, some of the observed traits are specific for Family 2 (Table 1, Fig. 2B and Supplementary material). Opposite to the short stature and overall present dysmorphic features seen in Family 1, affected Family 2 members present with a tall and thin stature and more pronounced dysmorphisms in males. The absence of the cleft palate in Family 2 members is another distinctive feature. The detected GABRA3 variant c.497C > T, p.T166M (Fig. 2C) affects a highly conserved amino acid in the Nterminal sequence of the GABA $\mathrm{A}$ receptor $\alpha_{3}$-subunit (Fig. 2D) and is predicted to be deleterious by in silico analysis. While it is not present in ExAC, one case has now been reported in gnomAD with a frequency of $5.61 \times 10^{-6}$ (Supplementary Table 1). To verify that no additional pathogenic autosomal variants were present in this family, whole exome sequencing was performed on Individuals II:1. III:3 III:6, III:7, III:10, III:12, and III:13 with no other genes emerging as directly related to the disease phenotype or explaining the inheritance pattern (Supplementary Table 3).

We further aimed to identify copy number variants involving GABRA3 by analysing a cohort of patients affected by epilepsy and intellectual disability $(n=103)$, only epilepsy $(n=198)$ or intellectual disability without epilepsy $(n=299)$, who underwent diagnostic high-density arrayCGH screening. An intragenic microduplication encompassing exons 1-3 of GABRA3 (chrX:152277607-152451201, GRCh38/hg38; $\operatorname{arr}[\mathrm{hg} 38]$ Xq28(152.277.607-152.451.201) $\mathrm{x} 2$ ) was identified in a male affected by pharmacoresistant epilepsy with weekly generalized seizures, generalized spikeand polyspike-wave discharges in EEG, but without any dysmorphic signs or nystagmus (Family 3) (Fig. 3A, C, Table 1 and Supplementary material). The duplication was inherited from a healthy mother. No deletions or duplications affecting GABRA3 were identified in a cohort of 273 in-house controls. In addition, no copy number variants encompassing the coding region of GABRA3 have been reported in the database of genomic variants (DGV database). We demonstrated that the rearrangement disrupts the expression of the gene in cultured fibroblasts from the patient, providing additional evidence for a pathogenic loss-of-function GABRA3 variant (Fig. 3B). Furthermore, whole exome sequencing was performed in this family to exclude any other potentially pathogenic variants, in particular those 


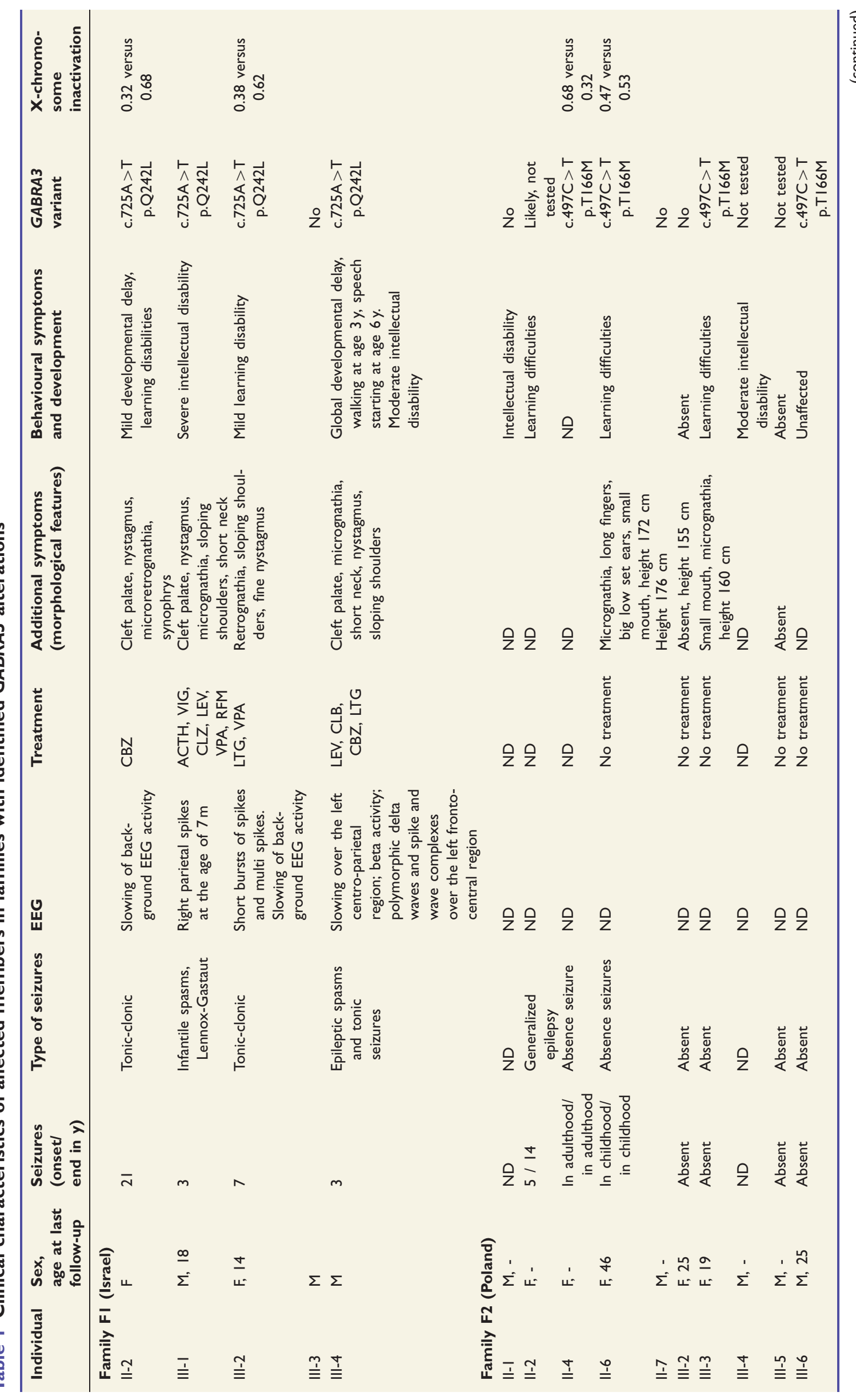




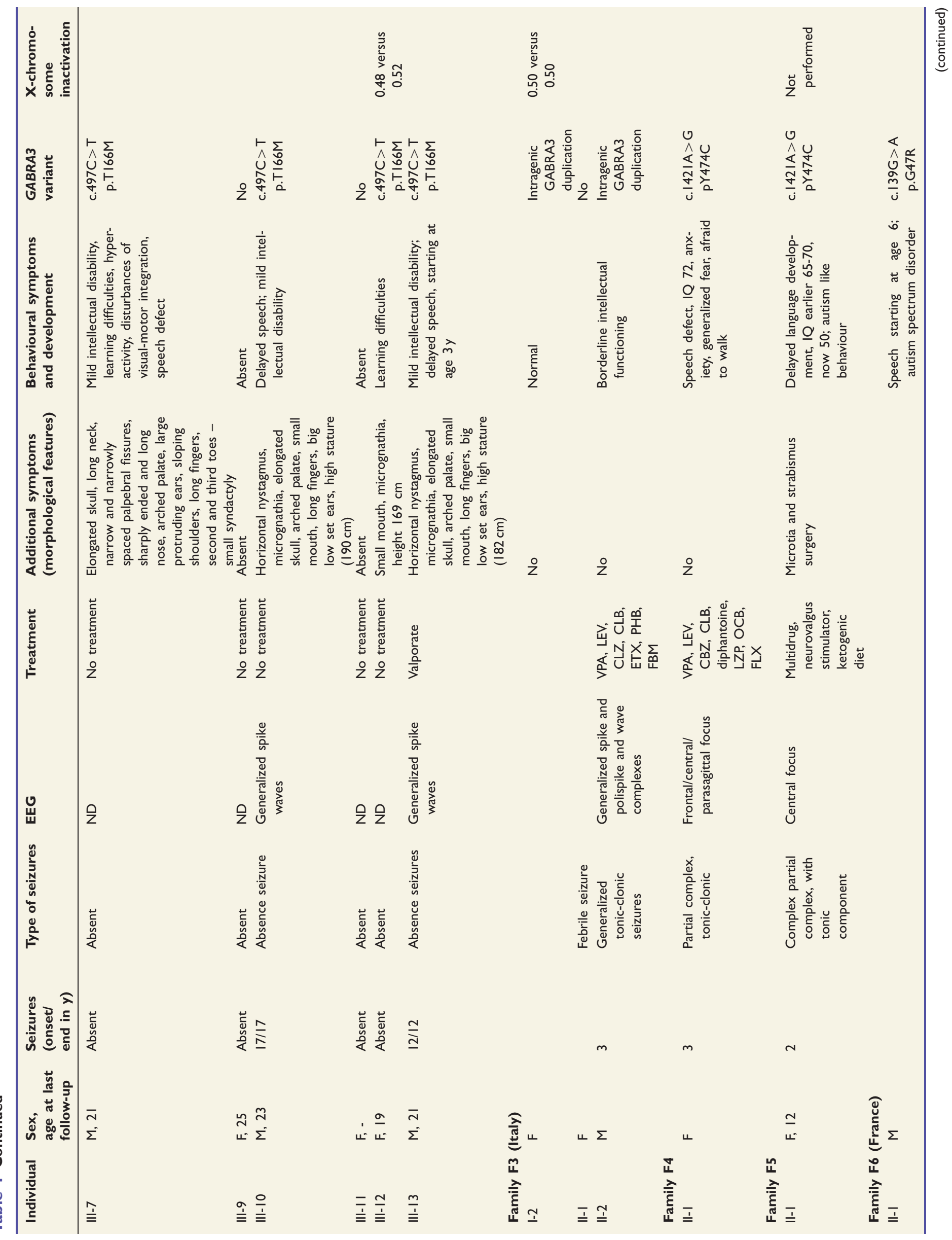


occurring de novo, which could explain the observed severe phenotype. However, no other genetic defect likely to contribute to the disease phenotype could be detected (Supplementary Table 4).

While the genetic analysis of these three families provided evidence for the co-segregation with the complex disease phenotype, we further explored the role of de novo occurring GABRA3 variants. Diagnostic trio-based whole exome sequencing revealed a recurrent de novo variant, p.Y474C, c.1421A $>\mathrm{G}$, in two sporadic females (Families 4 and 5) (Fig. 4A) presenting with partial seizures and mild-to-moderate intellectual disability. This variant affects transmembrane segment 4 of the receptor subunit and the replaced residue is highly conserved (Fig. 4B). The variant is not present in any of the control databases.

One more GABRA3 variant (c.139G >A, p.G47R) was identified in the $\mathrm{X}$-chromosome exome-sequencing study (Family 6) (Table 1 and Fig. 5A). The male proband presented with autism spectrum disorder and severe learning disabilities, but no epileptic seizures. His brother, who does not carry the variant, is affected with a very similar phenotype (Table 1 and Fig. 5A). This variant is found at a somewhat less conserved part of the $\mathrm{N}$-terminus of the $\alpha_{3}$-subunit (Fig. 5B). It has been identified in one male individual (allele frequency of $1.177 \times 10^{-5}$ in ExAC) and in two individuals in gnomAD (allele frequency $1.163 \times 10^{-5}$ ) (Supplementary Table 1). Furthermore, a different gene, $S L C 7 A 3$, has been proposed to cause the phenotype in both brothers of this family (Nava et al., 2015).

We further searched for pathogenic variants in GABRA3 in a cohort of 238 independent families with classical GGE syndromes that underwent whole exome sequencing. In a female proband (Family 7, Fig. 5C), suffering from GGE with generalized tonic-clonic seizures (EGTCS), we identified one further missense variant (c.1007C > T, p.T336M) affecting a conserved threonine in the extracellular loop between transmembrane segments 2 and 3 (Fig. 5D). Her unaffected mother also carried the variant, in contrast to her affected sister (suffering from classical childhood absence epilepsy) and her affected father (experiencing few generalized tonic-clonic seizures). Moreover, in this family there were no signs of additional dysmorphic features as observed in the first two families (Supplementary material and Table 1).

Finally, to test for a significant enrichment of GABRA3 variants in our cohorts compared to controls, we compared the allele frequency of the non-synonymous $G A B R A 3$ variants in our patient cohorts of the known size-excluding the diagnostic services-(4/733) with missense, nonsense and splice site variants reported in the ExAC Browser (84/87765). This calculation showed a significant enrichment of GABRA3 variants in the patient cohort using Fisher's exact test $(P<0.01)$.

Three out of the five missense variants are localized at the N-terminus of the $\alpha_{3}$-subunit (Fig. 6A), including the largely co-segregating variants from Families 1 (p.Q242L) and 2 (p.T166M) as well as the one found in the boy with 


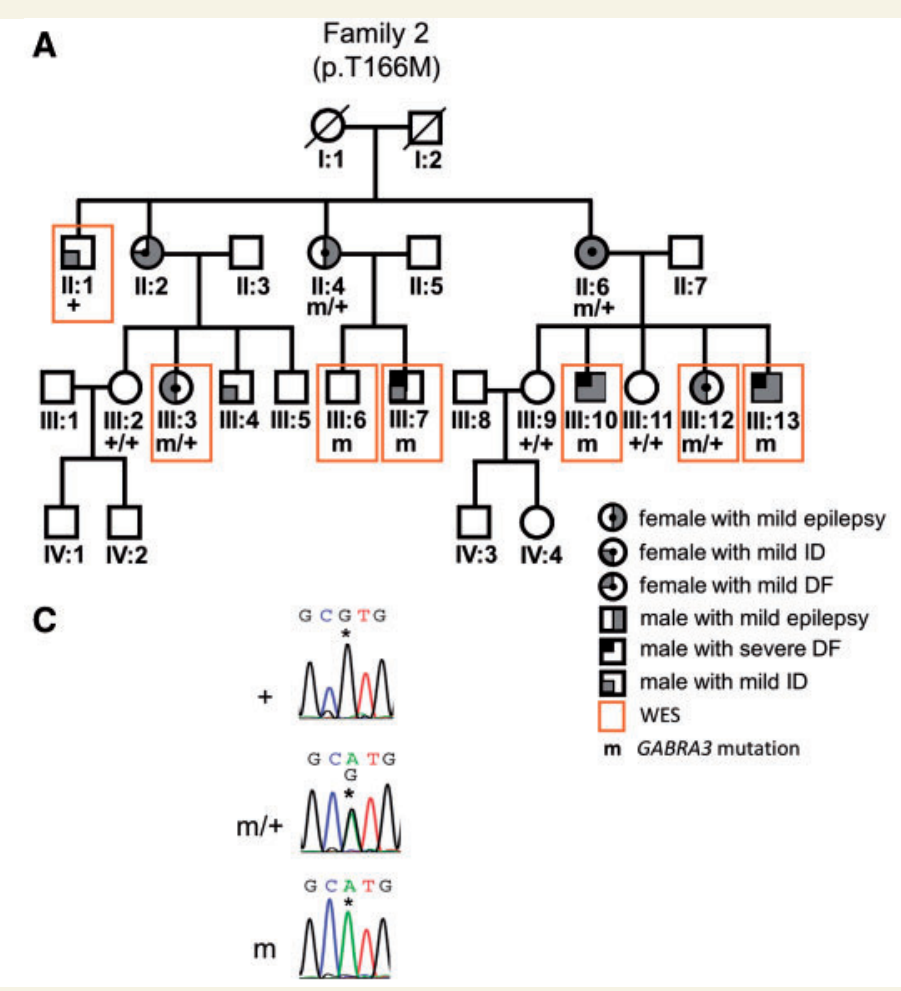

\section{B}

II:6

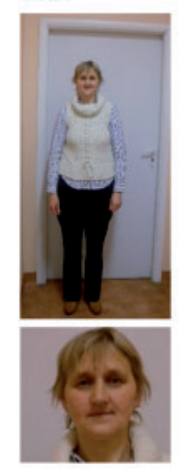

D

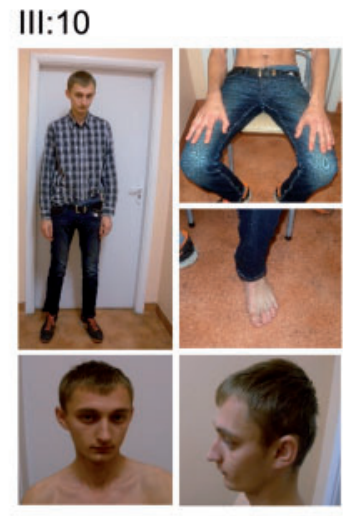

T166

HNMTTPNKL

HNMTTPNKI

HNMTTPNKI

HNMTTPNKI

HNMTTPNKI

HNMTMPNKI

HNMTMPNKI

HNMTAPNKI

HNMTTPNKI

HNMTTPNKI

Figure 2 Novel GABRA3 likely pathogenic variant associated with severe epilepsy. (A) Pedigree of Family 2 with co-segregation of the p.TI66M variant. (B) photographs of Patients II-6, III-I 0 and III-I 3 from Family 2, carrying the p. TI 66M variant. Micrognathia, elongated skull, small mouth, long fingers, big low set ears and high stature can be observed. (C) Sanger sequence chromatograms showing the genomic $\mathrm{G}>\mathrm{A}$ exchange. (D) p.TI66M amino acid exchange and the evolutionary conservation of the mutated amino acid. DF = dysmorphic features; ID = intellectual disability; WES = whole exome sequencing.

autism spectrum disorder (Family 6, p.G47R). The GGEassociated variant p.T336M affects the extracellular loop between transmembrane segments M2 and M3, whereas the de novo variant p.Y474C lies within the transmembrane segment M4 (Fig. 6A). We used Xenopus laevis oocytes and an automated two-microelectrode voltage clamp technique to assess the functional consequences of all identified variants. They were introduced in the cDNA encoding the human isoform of the $\mathrm{GABA}_{\mathrm{A}}$ receptor $\alpha_{3}$-subunit. After in vitro transcription, cRNAs encoding wild-type or mutant were co-injected with wild-type $\beta_{2}$ - and $\gamma_{2 s}$-subunits into oocytes and GABA-evoked ionic currents were recorded. We first investigated the effects of a high GABA concentration of $1 \mathrm{mM}$, closely mimicking physiological conditions when GABA is released into the synaptic cleft (Roth and Draguhn, 2012). This screening experiment revealed that all mutations, except p.G47R, led to a strong, statistically significant $(* * * P<0.0001$ ANOVA on ranks, Dunn's post hoc test) reduction of GABA-evoked currents compared to the wild-type (Fig. 6B and C). For p.Q242L, the currents were reduced by $85 \pm 3 \%$, for p.T166M by $75 \pm 3 \%$, for p.Y $474 \mathrm{C}$ by $68 \pm 9 \%$, for p.G47R by $46 \pm 10 \%$ and for p.T336M by $91 \pm 2 \%$ (Fig. 6B and C). Further investigation showed diminished responses to different GABA concentrations in the whole concentration range (Fig. 6D). Interestingly, the GABA sensitivity was increased for p.Q242L, p.Y474C and p.T336M variants compared to the wild-type $\left(\mathrm{EC}_{50}\right.$ of $25 \pm 2 ; 22 \pm 7$; $38 \pm 4$ and $96 \pm 3 \mu \mathrm{M}$ for mutants and wild-type, respectively). However, this shift was not predicted to compensate for the pronounced decline of current amplitudes (Fig. 6D). Western blot analysis of oocytes injected with wild-type or mutant $\alpha_{3} \beta_{2} \gamma_{2 s}$ receptors performed using an anti- $\alpha_{3}$-subunit antibody revealed a statistically significant decrease in the total amount of protein only for p.T166M compared to the wild-type (Fig. $6 \mathrm{E}$ and $\mathrm{F})\left({ }^{*} P<0.05\right.$, ANOVA on ranks, Dunn's post hoc test).

\section{Discussion}

Our results suggest that GABRA3 is a new gene associated with epilepsy and related disorders. In total, this study identified five missense variants and a microduplication within this gene. We provide strong genetic evidence for the pathogenicity of the fully co-segregating variant detected in Family 1. Family 2 is more complex, and the variant detected in this family, which largely co-segregated with epilepsy-related phenotypes in male and female carriers, is also found in an unaffected male, and one male affected only with intellectual disability. Especially Family 1 supports the pattern of X-linked inheritance, with males 
being more severely affected than females. This inheritance pattern also fits Family 3, since the boy in this family carrying a duplication with deleterious effects on GABRA3 expression also presented with severe epileptic
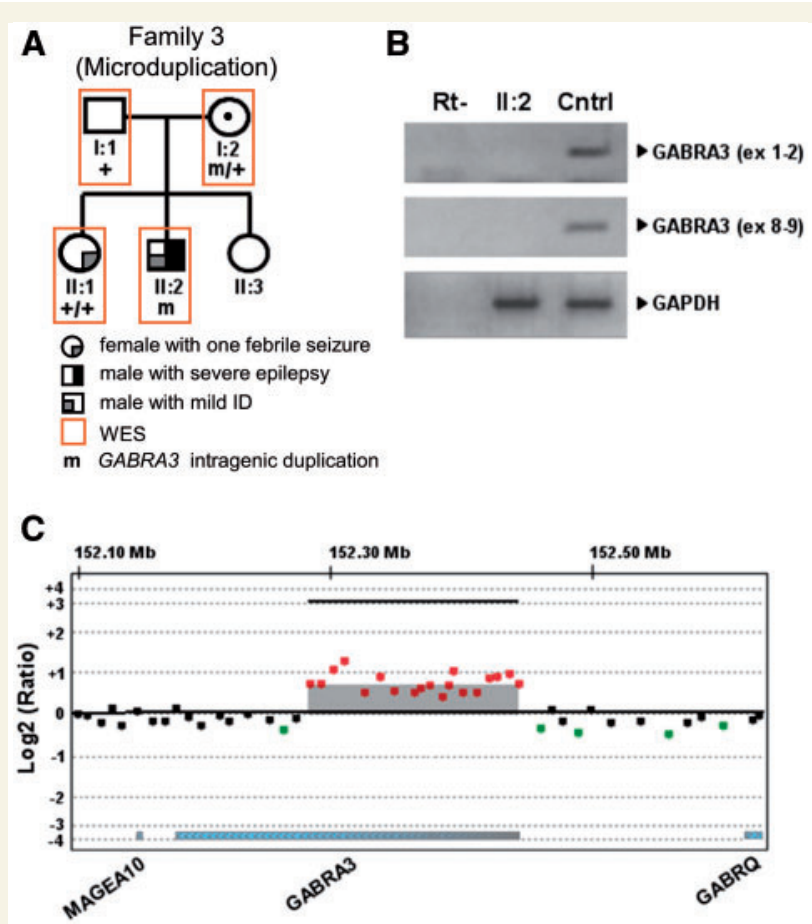

Figure 3 GABRA3 microduplication. (A) Pedigree of Family 3 revealing the co-segregation of the GABRA3 microduplication.

(B) RT-PCR showing that retro-transcribed GABRA3 RNA (exons I2 and exons 8-9) from cultured fibroblasts of the proband could not be detected; GAPDH was used as a housekeeping gene control.

(C) Plot of the Log2 intensity ratios showing amplified signals for probes spanning the 5' genomic region of GABRA3. The gene is displayed in the reverse strand. ID = intellectual disability; WES $=$ whole exome sequencing. encephalopathies in contrast to his unaffected mother. The detection of a recurrent de novo variant associated with a severe epileptic encephalopathies phenotype in females corroborates a role of this gene in epilepsy and related disorders. Moreover, in vitro analysis revealed that the severely affected individuals carried variants with a large functional deficit corresponding to at least a $70 \%$ reduction of GABA-evoked currents.

We also report two families in which GABRA3 cannot be regarded as an obvious disease gene, but might act as a risk factor contributing to the development of disease in some mutation carriers. This is seen both in the patient with autism spectrum disorder (Family 6) and in the female with mild epilepsy (Family 7 with GGE). In both families, clearly affected individuals do not carry GABRA3 variants, suggesting a dominant role of a different gene. In Family 6, this gene has already been suggested (Nava et al., 2015). Moreover, the GABRA3 variant detected in Family 6 showed a milder functional defect. The p.T336M amino acid exchange, which did show a very severe loss of function, was only found in two females within the GGE family, the unaffected mother and one of the affected sisters, but not in the other affected sister and the affected father. The incomplete co-segregation, as well as the fact that female mutation carriers from other families do not present with classical GGE syndromes, suggest that further factors or variants are involved in the pathogenesis within this family. However, these could not be identified by exome sequencing of the whole family (Supplementary Table 3).

The detected variants are associated with a large spectrum of neuropsychiatric symptoms, ranging from severe epileptic encephalopathies with dysmorphic features and nystagmus to relatively mild intellectual disability without epilepsy and one unaffected individual among the male mutation carriers. In females, the phenotypic spectrum extends
A
Family 4
(p.Y474C)
Family 5
B
(p.Y474C)
Y474

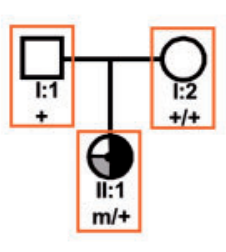
๑

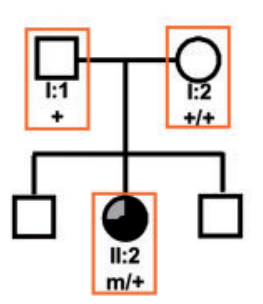
emale with severe epilepsy and severe ID
Female with mild ID
J Female with mild DF
WES
GABRA3 mutation

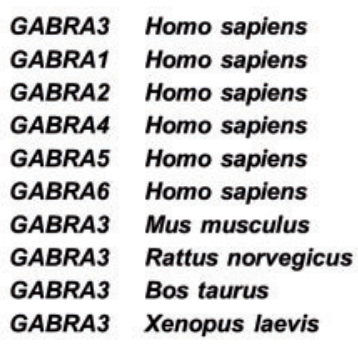
FNLVYWATY
FNLVYWATY
FNLVYWATY
FNMV YWVVY
FNLVYWATY
FNLVYWVVY
FNLVYWATY
FNLVYWATY
FNLVYWATY

Figure 4 Recurrent de novo GABRA3 variant identified in two unrelated girls. (A) Pedigrees of Families 4 and 5 showing the affected patients. (B) p.Y474C amino acid exchange and the evolutionary conservation of the mutated amino acid. DF = dysmorphic features; ID = intellectual disability; WES = whole exome sequencing. 
A

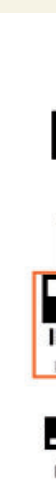

C

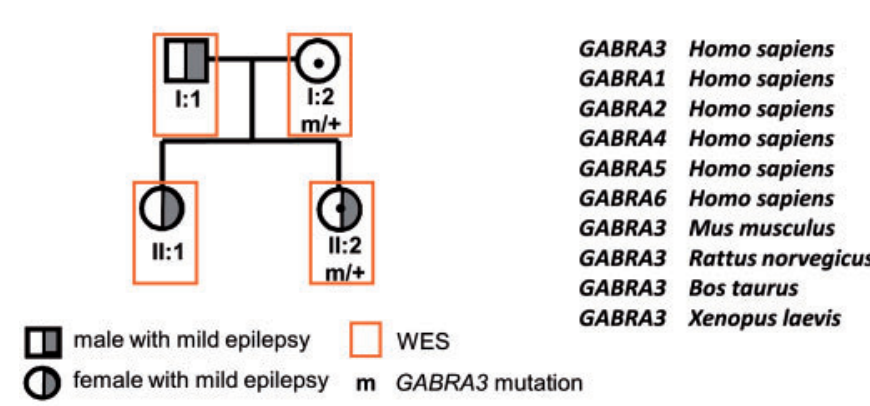

Family 7

(p.T336M)

B

(p.G47R)

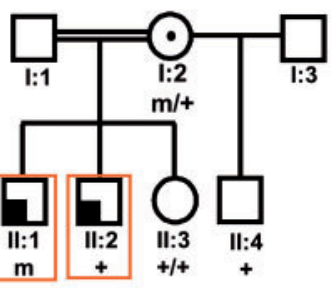

male with severe ID

m GABRA3 mutation

\section{(p.T336M)}

male with mild epilepsy

female with mild epilepsy m GABRA3 mutation

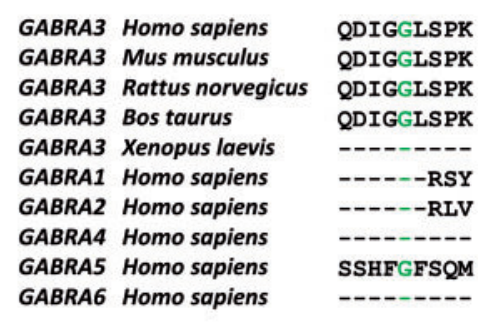

D

T336

Figure 5 GABRA3 variants identified in Family 6 with autism-like disorder and in Family 7 with GGE. (A) Family 6 pedigree showing the presence of the p.G47R $\alpha_{3}$ variant in the index case who presented with speech delay, autism spectrum disorder or behavioural disturbances and his mother. (B) Alignment depicting the evolutionary conservation of the p.G47 amino acid. (C) Family 7 pedigree showing the co-segregation of the P.T336M variant with the phenotype in this GGE family. (D) Alignment depicting the evolutionary conservation of the p.T336 amino acid. ID = intellectual disability; WES = whole exome sequencing.

from normal, i.e. without detectable clinical signs for epilepsy, intellectual disability or dysmorphisms, to mildly affected with well treatable seizures, moderate developmental delay and similar dysmorphic features and nystagmus as in males. This spectrum is further extended to the severe end in one of the de novo mutation carriers presenting with pharmacoresistant seizures in addition to moderate intellectual disability. We hypothesized that variable X-inactivation may contribute to the large phenotypic variability in females, but an X-inactivation test performed in leucocyte DNA from eight mutation carriers (affected $n=4$, unaffected $n=4)$ did not identify any correlation with the affection status (Table 1). However, the X-inactivation pattern in the nervous system can differ from that seen in blood, as reported for Rett syndrome (Gibson et al., 2005), so that we cannot exclude that $X$-inactivation plays a role in the phenotypic variability observed in female mutation carriers.

A broad phenotypic spectrum is well known from other epilepsy genes including those coding for different $\mathrm{GABA}_{\mathrm{A}}$ receptor subunits (Harkin et al., 2007; Carvill et al., 2014; Epi4K Consortium, 2016; Johannesen et al., 2016; Papandreou et al., 2016; Møller et al., 2017). Our results suggest that a combination of the severity of the mutation- induced $\mathrm{GABA}_{\mathrm{A}}$ receptor dysfunction and the 'genetic background', i.e. so far unknown modifying genetic factors that could not be identified in our exome sequencing studies, are responsible for the observed clinical phenotypic variability. The latter may play a role for example in the two different branches of Family 2 presenting males with large differences in clinical severity.

Some GABRA genes have further been linked to severe forms of epilepsy, such as Dravet syndrome (GABRA1) (Carvill et al., 2014; Johannesen et al., 2016), or LennoxGastaut syndrome (GABRB3) (Epi4K Consortium et al., 2013). Genetic studies have shown that many of the most severe epileptic encephalopathies are caused by de novo mutations (Claes et al., 2001; Harkin et al., 2002; Kalscheuer et al., 2003; Tao et al., 2004; Veeramah et al., 2012; Weckhuysen et al., 2012; Epi4K Consortium et al., 2013; Suls et al., 2013; Carvill et al., 2014; Nava et al., 2014; Blanchard et al., 2015; Syrbe et al., 2015; Epi4K Consortium, 2016; Johannesen et al., 2016; Shen et al., 2017), which is confirmed by our findings in one of the de novo mutation carriers. However, our results also indicate that mildly affected female carriers can transfer severe mutations to their children, which can result in devastating epileptic encephalopathies and intellectual disability in 
A

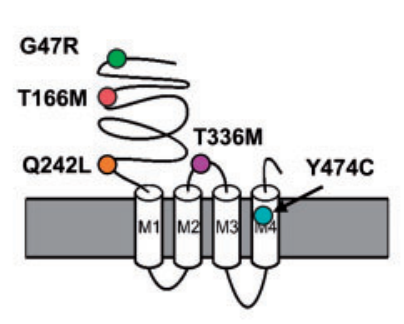

C

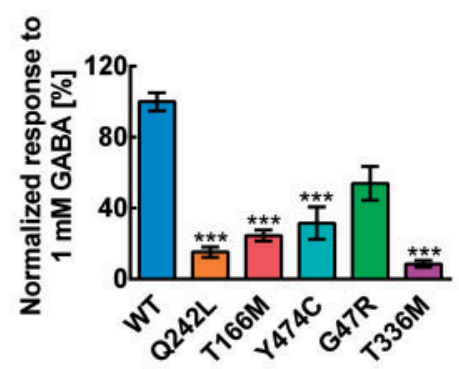

E

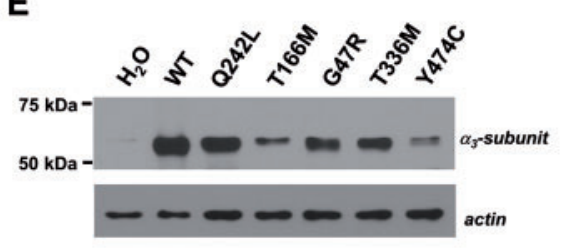

D
B
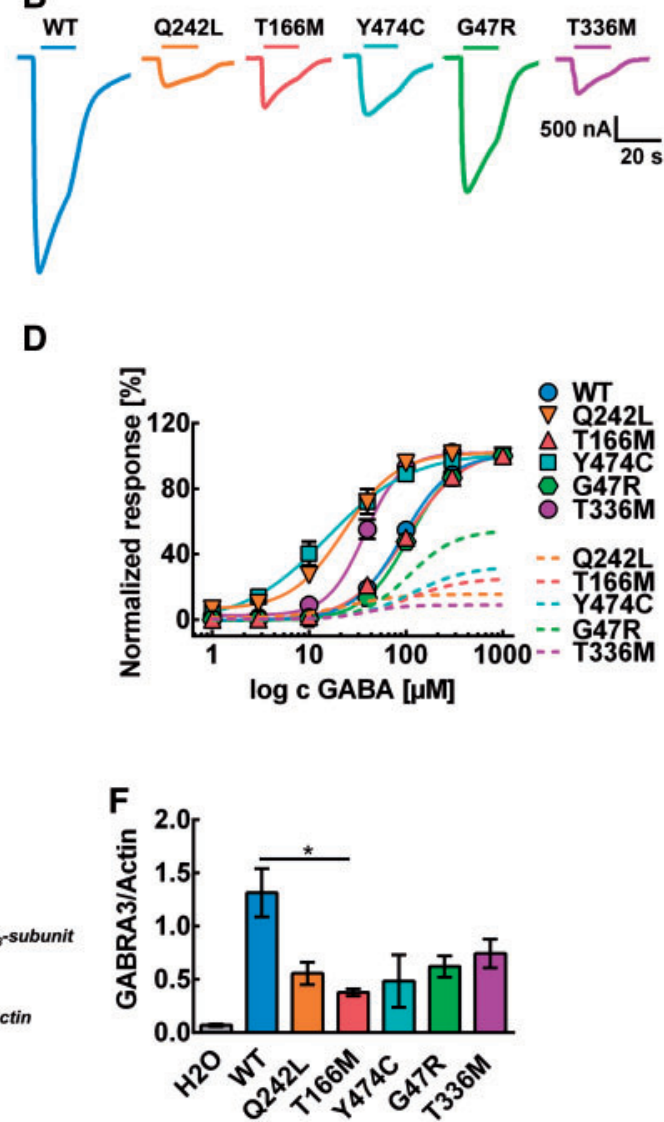

Figure 6 Functional analysis of the identified GABRA3 variants in Xenopus laevis oocytes. (A) Schematic representation of the $\alpha_{3}$ subunit of the $\mathrm{GABA}_{A}$ receptor including the predicted positions of all mutated amino acids. (B) Representative examples of current responses to application of I mM GABA recorded from Xenopus oocytes expressing $\alpha_{3} \beta_{2} \gamma_{2 s}$ receptors comprising wild-type or mutant (p.Q242L, p.TI66M, p.Y474C, p.G47R, p.T336M) $\alpha_{3}$-subunits. (C) Normalized current response to I mM GABA application for wild-type $(n=137)$, p.Q242L ( $\left.n=29\right)$, p.TI66M ( $n=37)$, p.Y474C $(n=22)$, p.G47R $(n=10)$ and P.T336M $(n=28)$ mutations; ***P $<0.000$ I ANOVA on ranks, Dunn's post hoc test. The reduction in current amplitude was $85 \pm 3 \%$ for p.Q242L; $75 \pm 3 \%$ p.TI66M; $68 \pm 9 \%$ for p.Y474C; $46 \pm 10 \%$ for p.G47R and 9 I $\pm 2 \%$ for p.T336M in comparison with the wild-type. (D) Dose-response curve for $\alpha_{3} \beta_{2} \gamma_{2 s}$ wild-type $(n=77)$, p.Q242L $(n=16)$, p.TI66M $(n=25)$, p.Y474C $(n=9)$, p.G47R $(n=19)$ and P.T336M $(n=14)$ receptors recorded upon application of different GABA concentrations (in $\mu$ M: I, 3, 10, 40, I00, 300 and $1000)$ and normalized to the maximal response $(1000 \mu \mathrm{M})$ for each cell. Statistically significant differences between the $\mathrm{EC}_{50}$ values were verified by ANOVA on ranks and Dunn's post hoc test (***P $<0.000$ I for p.Q242L, p.Y474C and p.T336M). The predicted dose-response curves, calculated from the current amplitude ratio of the mutant versus wild-type response to the application of I mM GABA (see $\mathbf{C}$ ) are shown as dashed lines for all mutations. (E) Western blot analysis of whole cell lysates from Xenopus oocytes injected with cRNA encoding for $\alpha_{3} \beta_{2} \gamma_{2 s}$ wild-type or mutated subunits. All lysates show a band of the expected size for the $\alpha_{3}$-subunit protein $(55 \mathrm{kDa})$. (F) Quantification of western blots revealing a significant reduction of the $\alpha_{3}$-subunit signal for the TI66M mutant $(n=4) .{ }^{*} P<0.05$ using ANOVA on ranks with Dunn's post hoc test.

the sons and therefore constitutes an important aspect of genetic counseling in these families with X-linkedlike inheritance.

Reduced amplitudes of GABA-evoked currents were observed for all five detected variants. This loss-of-function effect has been a common feature of all $\mathrm{GABA}_{\mathrm{A}}$ receptor mutations associated with epilepsy and proposed to impair the $\mathrm{GABA}_{\mathrm{A}}$ receptor-mediated inhibition leading to an increased neuronal hyperexcitability and seizures (Baulac et al., 2001; Wallace et al., 2001; Harkin et al., 2002; Maljevic et al., 2006; Johannesen et al., 2016; Møller et al., 2017). The suggested molecular mechanisms include gating defects, reduced GABA sensitivity, or reduced surface expression due to protein misfolding, impaired assembly or trafficking defects (Wallace et al., 2001; Harkin et al., 2002; Maljevic et al., 2006; Macdonald et al., 2012; Johannesen et al., 2016; Kang and Macdonald, 2016; Møller et al., 2017). Messenger RNA and protein degradation of defective $\mathrm{GABA}_{\mathrm{A}}$ receptor subunits have also been shown (Gallagher et al., 2005; Maljevic et al., 2006; Kang and Macdonald, 2016). In our study, only one (p.T166M) of the five GABRA3 variants showed a significantly lower amount of protein in total lysates obtained from injected Xenopus oocytes, indicating that at least for 
this variant a less stable protein is the cause for the observed reduced current amplitudes.

Interestingly, three of the analysed five variants showed an increased GABA sensitivity. Since the amplitudes of GABA-evoked anion currents carried by the respective mutant receptors were strongly diminished, we estimated the combined effect of the amplitude reduction and the shift in GABA-sensitivity, which revealed that the effect of this shift may only have a minor gain-of-function effect at low GABA concentrations. At higher GABA concentrations, such as $1 \mathrm{mM}$, which is considered to represent the GABA concentration in the synaptic cleft (Roth and Draguhn, 2012), a severe loss-of-function remained obvious. However, this repeatedly identified functional alteration may suggest that other complex mechanisms, possibly including pre- or extrasynaptic processes may underlie pathology associated with the $\alpha_{3}$-subunit variants. The role of the $\alpha_{3}$-subunit in tonic inhibition has been demonstrated in principal cells of the amygdala (Marowsky et al., 2012). Furthermore, the fact that $\mathrm{GABA}_{\mathrm{A}}$ receptors containing $\alpha_{3}$-subunits show a higher affinity for GABA compared to those containing $\alpha_{1}$-subunits (Keramidas and Harrison, 2010) might also indicate their extrasynaptic role.

In the rodent brain, the $\alpha_{3}$-subunit of the $\mathrm{GABA}_{\mathrm{A}}$ receptor has a broad distribution and is specifically expressed in the thalamus, being the only $\alpha$-subunit expressed in the nucleus reticularis thalami (nRT) (Pirker et al., 2000; Hörtnagl et al., 2013). Knockout mice lacking this subunit do not show spontaneous seizures. On the contrary, they show an enhanced intra nucleus reticularis thalami inhibition and a reduced susceptibility to pharmacologically induced seizures, probably due to a strong compensatory mechanism increasing the expression of a different $\alpha$-subunit (Schofield et al., 2009). However, in a mouse model in which the benzodiazepine binding site of the $\alpha_{3}$-subunit is disrupted, an increase in spike and wave discharges, a characteristic of absence seizures, was found (Christian et al., 2013). This suggests that an impaired function of the $\alpha_{3^{-}}$ subunit may lead to epileptic seizures, which are not detected in knockout animals because of an adaptive process or compensatory mechanism.

Dysmorphic features are observed in the two families with the most severe phenotypes in most affected mutation carriers and partially overlap between the two families. Also one of the de novo mutation carriers showed a (different) dysmorphism. How a subunit of $\mathrm{GABA}_{\mathrm{A}}$ receptors may be linked to changes in morphology is not clear, although it has been reported that homozygous Gabrb3 knockout mice exhibit a cleft palate in about half of the cases and the other half have feeding difficulties as neonates (DeLorey et al., 1998). The observed nystagmus may be attributed to a particular inhibitory function of $\mathrm{GABA}_{\mathrm{A}}$ receptor $\alpha_{3}$-subunits in the brainstem or cerebellum. Expression of GABRA3 in these structures has been shown using specific antibodies against this subunit (Pirker et al., 2000).
In summary, we have detected GABRA3 variants in patients with a spectrum of neuropsychiatric disorders and demonstrated in our in vitro assay that they cause lossof-function effects. The data obtained from whole exome sequencing in the affected families suggest that GABRA3 variants present the major underlying genetic component of the observed disease phenotype in three and a contributing factor in two families. Detection of a recurrent de novo variant associated with a severe clinical picture corroborates these findings suggesting GABRA3 as a new epilepsy gene.

\section{Acknowledgements}

We dedicate this work to our colleague Esther LeshinskySilver who initiated the study by identifying the first GABRA3 mutation and sadly passed away during the reviewing process of this manuscript.

We thank all the families who participated in this research and Prof. S. Schweiger for her critical reading, Prof. S. Petrou for providing the GABRA3 clone, Heidi Loeffler and Nicole Zepezauer for excellent technical assistance. Some of the computational results presented in this paper were carried out using the HPC facilities of the University of Luxembourg (http://hpc.uni.lu).

\section{Funding}

Parts of this work were financed by the EU FP7 project GENCODYS, grant number 241995, by the Polish Ministry of Science and Higher Education, grant number 2014/15/D/NZ5/03426, by the Italian Ministry of Health (project RF-2010-2314356 to F.Z. and GR-2009-1473821 to P.S.), by the European Science Foundation Eurocores project EuroEPINOMICS (CoGIE) (DFG Le1030/11-1 to H.L. and S.M.), and by the Federal Ministry for Education and Research (BMBF, program on rare diseases, IonNeurONet: 01GM1105A, to S.M., H.L., S.B.).

\section{Supplementary material}

Supplementary material is available at Brain online.

\section{References}

Genomes Project Consortium, Abecasis GR, Auton A, Brooks LD, DePristo MA, Durbin RM, et al. An integrated map of genetic variation from 1,092 human genomes. Nature 2012; 491: 56-65.

Baulac S, Huberfeld G, Gourfinkel-An I, Mitropoulou G, Beranger A, Prud'homme JF, et al. First genetic evidence of GABA(A) receptor dysfunction in epilepsy: a mutation in the gamma2-subunit gene. Nat Genet 2001; 28: 46-8.

Blanchard MG, Willemsen MH, Walker JB, Dib-Hajj SD, Waxman $\mathrm{SG}$, Jongmans MC, et al. De novo gain-of-function and loss-of-function mutations of SCN8A in patients with intellectual disabilities and epilepsy. J Med Genet 2015; 52: 330-7. 
Carvill GL, Weckhuysen S, McMahon JM, Hartmann C, Møller RS, Hjalgrim $\mathrm{H}$, et al. GABRA1 and STXBP1: novel genetic causes of Dravet syndrome. Neurology 2014; 82: 1245-53.

Christian CA, Herbert AG, Holt RL, Peng K, Sherwood KD, PangratzFuehrer S, et al. Endogenous positive allosteric modulation of GABA(A) receptors by diazepam binding inhibitor. Neuron 2013; 78: 1063-74.

Claes L, Del-Favero J, Ceulemans B, Lagae L, Van Broeckhoven C, De Jonghe P. De novo mutations in the sodium-channel gene SCN1A cause severe myoclonic epilepsy of infancy. Am J Hum Genet 2001; 68: $1327-32$.

Coppola A, Striano P, Gimelli S, Ciampa C, Santulli L, Caranci F, et al. A de novo $11 \mathrm{p} 12-\mathrm{p} 15.4$ duplication in a patient with pharmacoresistant epilepsy, mental retardation, and dysmorphisms. Brain Dev 2010; 32: 248-52.

Cossette P, Liu L, Brisebois K, Dong H, Lortie A, Vanasse M, et al. Mutation of GABRA1 in an autosomal dominant form of juvenile myoclonic epilepsy. Nat Genet 2002; 31: 184-9.

DeLorey TM, Handforth A, Anagnostaras SG, Homanics GE, Minassian BA, Asatourian A, et al. Mice lacking the beta3 subunit of the GABAA receptor have the epilepsy phenotype and many of the behavioral characteristics of Angelman syndrome. J Neurosci 1998; 18: 8505-14.

DePristo MA, Banks E, Poplin R, Garimella KV, Maguire JR, Hartl C, et al. A framework for variation discovery and genotyping using next-generation DNA sequencing data. Nat Genet 2011; 43: 491-8.

Epi4K Consortium, Epilepsy Phenome/Genome Project, Allen AS, Berkovic SF, Cossette P, Delanty N, et al. De novo mutations in epileptic encephalopathies. Nature 2013; 501: 217-21.

Epi4K Consortium. Electronic address: epi4k@columbia.edu, Epi4K Consortium. De Novo Mutations in SLC1A2 and CACNA1A are important causes of epileptic encephalopathies. Am J Hum Genet 2016; 99: 287-98.

Fritschy JM, Panzanelli P. GABAA receptors and plasticity of inhibitory neurotransmission in the central nervous system. Eur J Neurosci 2014; 39: 1845-65.

Gallagher MJ, Shen W, Song L, Macdonald RL. Endoplasmic reticulum retention and associated degradation of a GABAA receptor epilepsy mutation that inserts an aspartate in the M3 transmembrane segment of the alpha1 subunit. J Biol Chem 2005; 280: 379958004.

Gibson JH, Williamson SL, Arbuckle S, Christodoulou J. X chromosome inactivation patterns in brain in Rett syndrome: implications for the disease phenotype. Brain Dev 2005; 27: 266-70.

Harkin LA, Bowser DN, Dibbens LM, Singh R, Phillips F, Wallace $\mathrm{RH}$, et al. Truncation of the GABA(A)-receptor gamma2 subunit in a family with generalized epilepsy with febrile seizures plus. Am J Hum Genet 2002; 70: 530-36.

Harkin LA, McMahon JM, Iona X, Dibbens L, Pelekanos JT, Zuberi $\mathrm{SM}$, et al. The spectrum of SCN1A-related infantile epileptic encephalopathies. Brain 2007; 130: 843-52.

Hörtnagl H, Tasan RO, Wieselthaler A, Kirchmair E, Sieghart W, Sperk G. Patterns of mRNA and protein expression for 12 GABAA receptor subunits in the mouse brain. Neuroscience 2013; 236: 345-72.

Howell KB, McMahon JM, Carvill GL, Tambunan D, Mackay MT, Rodriguez-Casero V, et al. SCN2A encephalopathy: a major cause of epilepsy of infancy with migrating focal seizures. Neurology 2015; 85: 958-66.

Hu H, Haas SA, Chelly J, Van Esch H, Raynaud M, de Brouwer AP, et al. X-exome sequencing of 405 unresolved families identifies seven novel intellectual disability genes. Mol Psychiatry 2016; 21: 133-48.

$\mathrm{Hu} \mathrm{H}$, Wienker TF, Musante L, Kalscheuer VM, Kahrizi K, Najmabadi $\mathrm{H}$, et al. Integrated sequence analysis pipeline provides one-stop solution for identifying disease-causing mutations. Hum Mutat 2014; 35: 1427-35.
Janve VS, Hernandez CC, Verdier KM, Hu N, Macdonald RL. Epileptic encephalopathy de novo GABRB mutations impair GABAA receptor function. Ann Neurol 2017; 79: 806-25.

Johannesen K, Marini C, Pfeffer S, Møller RS, Dorn T, Niturad C, et al. Phenotypic spectrum of GABRA1: from generalized epilepsies to severe epileptic encephalopathies. Neurology 2016; 87: 1140-51.

Kalscheuer VM, Tao J, Donnelly A, Hollway G, Schwinger E, Kübart $\mathrm{S}$, et al. Disruption of the serine/threonine kinase 9 gene causes severe X-linked infantile spasms and mental retardation. Am J Hum Genet 2003; 72: 1401-11.

Kananura C, Haug K, Sander T, Runge U, Gu W, Hallmann K, et al. A splice-site mutation in GABRG2 associated with childhood absence epilepsy and febrile convulsions. Arch Neurol 2002; 59: 1137-41.

Kang JQ, Macdonald RL. Molecular pathogenic basis for GABRG2 mutations associated with a spectrum of epilepsy syndromes, from generalized absence epilepsy to Dravet syndrome. JAMA Neurol 2016; 73: 1009-16.

Keramidas A, Harrison NL. The activation mechanism of alpha1beta2gamma2S and alpha3beta3gamma2S GABAA receptors. J Gen Physiol 2010; 135: 59-75.

Kircher M, Witten DM, Jain P, O’Roak BJ, Cooper GM, Shendure J. A general framework for estimating the relative pathogenicity of human genetic variants. Nat Genet 2014; 46: 310-15.

Larsen J, Carvill GL, Gardella E, Kluger G, Schmiedel G, Barisic N, et al. The phenotypic spectrum of SCN8A encephalopathy. Neurology 2015; 84: 480-9.

Leen WG, Klepper J, Verbeek MM, Leferink M, Hofste T, van Engelen BG, et al. Glucose transporter-1 deficiency syndrome: the expanding clinical and genetic spectrum of a treatable disorder. Brain 2010; 133: 655-70.

Lek M, Karczewski KJ, Minikel EV, Samocha KE, Banks E, Fennell T, et al. Analysis of protein-coding genetic variation in 60,706 humans. Nature 2016; 536: 285-91.

Lemke JR, Hendrickx R, Geider K, Laube B, Schwake M, Harvey RJ, et al. GRIN2B mutations in West syndrome and intellectual disability with focal epilepsy. Ann Neurol 2014; 75: 147-54.

Lerche H, Shah M, Beck H, Noebels J, Johnston D, Vincent A. Ion channels in genetic and acquired forms of epilepsy. J Physiol 2013; 591: 753-64.

Leu C, Coppola A, Sisodiya SM. Progress from genome-wide association studies and copy number variant studies in epilepsy. Curr Opin Neurol 2016; 29: 158-67.

Li H, Durbin R. Fast and accurate short read alignment with BurrowsWheeler transform. Bioinformatics 2009; 25: 54-60.

Li H, Handsaker B, Wysoker A, Fennell T, Ruan J, Homer N, et al. The sequence alignment/map format and SAMtools. Bioinformatics 2009; 25: 2078-9.

Liu X, Jian X, Boerwinkle E. dbNSFP: a lightweight database of human nonsynonymous SNPs and their functional predictions. Hum Mutat 2011; 32: 894-9.

Liu X, Wu C, Li C, Boerwinkle E. dbNSFP v3.0: a one-stop database of functional predictions and annotations for human nonsynonymous and splice-site SNVs. Hum Mutat 2016; 37: 235-41.

Macdonald RL, Kang JQ, Gallagher MJ. GABAA receptor subunit mutations and genetic epilepsies. In: Noebels JL, Avoli M, Rogawski MA, Olsen RW, Delgado-Escueta AV, editors. Jasper's basic mechanisms of the epilepsies. Bethesda, MD: National Center for Biotechnology Information; 2012.

Maljevic S, Krampfl K, Cobilanschi J, Tilgen N, Beyer S, Weber YG, et al. A mutation in the GABA(A) receptor alpha(1)-subunit is associated with absence epilepsy. Ann Neurol 2006; 59: 983-7.

Marowsky A, Rudolph U, Fritschy JM, Arand M. Tonic inhibition in principal cells of the amygdala: a central role for $\alpha 3$ subunit-containing GABAA receptors. J Neurosci 2012; 32: 8611-19. 
Møller RS, Wuttke TV, Helbig I, Marini C, Johannesen KM, Brilstra $\mathrm{EH}$, et al. Mutations in GABRB3: from febrile seizures to epileptic encephalopathies. Neurology 2017; 88: 483-92.

Nava C, Dalle C, Rastetter A, Striano P, de Kovel CG, Nabbout R, et al. De novo mutations in HCN1 cause early infantile epileptic encephalopathy. Nat Genet 2014; 46: 640-5.

Nava C, Rupp J, Boissel JP, Mignot C, Rastetter A, Amiet C, et al. Hypomorphic variants of cationic amino acid transporter 3 in males with autism spectrum disorders. Amino Acids 2015; 47: 2647-58.

Papandreou A, McTague A, Trump N, Ambegaonkar G, Ngoh A, Meyer E, et al. GABRB3 mutations: a new and emerging cause of early infantile epileptic encephalopathy. Dev Med Child Neurol 2016; 58: 416-20.

Pirker S, Schwarzer C, Wieselthaler A, Sieghart W, Sperk G. GABA(A) receptors: immunocytochemical distribution of 13 subunits in the adult rat brain. Neuroscience 2000; 101: 815-50.

Reumers J, De Rijk P, Zhao H, Liekens A, Smeets D, Cleary J, et al. Optimized filtering reduces the error rate in detecting genomic variants by short-read sequencing. Nat Biotechnol 2011; 30: 61-8.

Roth FC, Draguhn A. GABA metabolism and transport: effects on synaptic efficacy. Neural Plast 2012; 2012: 805830.

Schofield CM, Kleiman-Weiner M, Rudolph U, Huguenard JR. A gain in GABAA receptor synaptic strength in thalamus reduces oscillatory activity and absence seizures. Proc Natl Acad Sci USA 2009; 106: 7630-5.

Schubert J, Siekierska A, Langlois M, May P, Huneau C, Becker F, et al. Mutations in STX1B, encoding a presynaptic protein, cause fever-associated epilepsy syndromes. Nat Genet 2014; 46: 1327-32.

Shen D, Hernandez CC, Shen W, Hu N, Poduri A, Shiedley B, et al. De novo GABRG2 mutations associated with epileptic encephalopathies. Brain 2017; 140: 49-67.

Sherry ST, Ward MH, Kholodov M, Baker J, Phan L, Smigielski EM, et al. dbSNP: the NCBI database of genetic variation. Nucleic Acids Res 2001; 29: 308-11.
Suls A, Jaehn JA, Kecskés A, Weber Y, Weckhuysen S, Craiu DC, et al. De novo loss-of-function mutations in CHD2 cause a feversensitive myoclonic epileptic encephalopathy sharing features with Dravet syndrome. Am J Hum Genet 2013; 93: 967-75.

Syrbe S, Hedrich UB, Riesch E, Djémié T, Müller S, Møller RS, et al. De novo loss- or gain-of-function mutations in KCNA2 cause epileptic encephalopathy. Nat Genet 2015; 47: 393-9.

Tao J, Van Esch H, Hagedorn-Greiwe M, Hoffmann K, Moser B, Raynaud $\mathrm{M}$, et al. Mutations in the X-linked cyclin-dependent kinase-like 5 (CDKL5/STK9) gene are associated with severe neurodevelopmental retardation. Am J Hum Genet 2004; 75: 1149-54.

Veeramah KR, O’Brien JE, Meisler MH, Cheng X, Dib-Hajj SD, Waxman SG, et al. De novo pathogenic SCN8A mutation identified by whole-genome sequencing of a family quartet affected by infantile epileptic encephalopathy and SUDEP. Am J Hum Genet 2012; 90: 502-10.

Wallace RH, Marini C, Petrou S, Harkin LA, Bowser DN, Panchal RG, et al. Mutant GABA(A) receptor gamma2-subunit in childhood absence epilepsy and febrile seizures. Nat Genet 2001; 28: 49-52.

Wang K, Li M, Hakonarson H. ANNOVAR: functional annotation of genetic variants from high-throughput sequencing data. Nucleic Acids Res 2010; 38: e164.

Weckhuysen S, Korff CM. Epilepsy: old syndromes, new genes. Curr Neurol Neurosci Rep 2014; 14: 447.

Weckhuysen S, Mandelstam S, Suls A, Audenaert D, Deconinck T, Claes LR, et al. KCNQ2 encephalopathy: emerging phenotype of a neonatal epileptic encephalopathy. Ann Neurol 2012; 71: $15-25$.

Wolff M, Johannesen KM, Hedrich UB, Masnada S, Rubboli G, et al. Genetic and phenotypic heterogeneity suggest therapeutic implications in SCN2A-related disorders. Brain 2017; 140: 1316-36. 